\title{
Neural correlates of body comparison and weight estimation in weight-recovered anorexia nervosa: a functional magnetic resonance imaging study
}

Naoki Kodama ${ }^{1 *}$ (D, Yoshiya Moriguchi², Aya Takeda ${ }^{3}$, Motonari Maeda $^{4}$, Tetsuya Ando ${ }^{5}$, Hiroe Kikuchi ${ }^{6}$, Motoharu Gondo ${ }^{7}$, Hiroaki Adachi ${ }^{8}$ and Gen Komaki ${ }^{9}$

\begin{abstract}
Background: The neural mechanisms underlying body dissatisfaction and emotional problems evoked by social comparisons in patients with anorexia nervosa (AN) are currently unclear. Here, we elucidate patterns of brain activation among recovered patients with AN (recAN) during body comparison and weight estimation with functional magnetic resonance imaging (fMRI).

Methods: We used fMRI to examine 12 patients with recAN and 13 healthy controls while they performed body comparison and weight estimation tasks with images of underweight, healthy weight, and overweight female bodies. In the body comparison task, participants rated their anxiety levels while comparing their own body with the presented image. In the weight estimation task, participants estimated the weight of the body in the presented image. We used between-group region of interest (ROI) analyses of the blood oxygen level dependent (BOLD) signal to analyze differences in brain activation patterns between the groups. In addition, to investigate activation outside predetermined ROls, we performed an exploratory whole-brain analysis to identify group differences.

Results: We found that, compared to healthy controls, patients with recAN exhibited significantly greater activation in the pregenual anterior cingulate cortex (pgACC) when comparing their own bodies with images of underweight female bodies. In addition, we found that, compared with healthy controls, patients with recAN exhibited significantly smaller activation in the middle temporal gyrus corresponding to the extrastriate body area (EBA) when comparing their own bodies, irrespective of weight, during self-other comparisons of body shape.

Conclusions: Our findings from a group of patients with recAN suggest that the pathology of AN may lie in an inability to regulate negative affect in response to body images via pgACC activation during body comparisons. The findings also suggest that altered body image processing in the brain persists even after recovery from AN.
\end{abstract}

Keywords: Body dissatisfaction, Body image, Anorexia nervosa, Anterior cingulate cortex, Extrastriate body area, fMRI

\footnotetext{
* Correspondence: kodama-n@med.uoeh-u.ac.jp

1 Division of Psychosomatic Medicine, Department of Neurology, University of

Occupational and Environmental Health, Kitakyushu 807-8555, Japan

Full list of author information is available at the end of the article
}

(c) The Author(s). 2018 Open Access This article is distributed under the terms of the Creative Commons Attribution 4.0 International License (http://creativecommons.org/licenses/by/4.0/), which permits unrestricted use, distribution, and reproduction in any medium, provided you give appropriate credit to the original author(s) and the source, provide a link to the Creative Commons license, and indicate if changes were made. The Creative Commons Public Domain Dedication waiver (http://creativecommons.org/publicdomain/zero/1.0/) applies to the data made available in this article, unless otherwise stated. 


\section{Background}

Anorexia nervosa (AN) is a disorder of unknown etiology, mostly affecting young women. AN is characterized by immoderate food restriction, inappropriate eating habits, and distorted body image. The condition is associated with high rates of chronicity, morbidity, and mortality. However, the lack of understanding of the pathophysiology underlying AN has hindered the development of effective treatments.

Body dissatisfaction is a core pathophysiological feature of $\mathrm{AN}$, manifesting as a negative subjective evaluation of the weight and shape of one's own body. Indeed, body dissatisfaction is considered a diagnostic feature of $\mathrm{AN}$, defined as "undue influence of weight and shape on self-evaluation" (American Psychiatric Association, 2013), and is considered an important factor in the development, maintenance, and relapse of AN [1-3].

The increasing prevalence of body dissatisfaction may be related to the sociocultural impact of the "thin ideal", by which the concept of an idealized slim female body is promoted by mass media [4]. Current societal standards of beauty promote the thin ideal, which most women are unable to achieve [5]. Furthermore, research has consistently shown that the extent of exposure to mass media is positively correlated with body dissatisfaction [4]. Exposure to idealized female bodies via mass media is thought to prompt viewers to compare their own body with the ideal body shape, resulting in dissatisfaction with their own body and consequent emotional distress or anxiety, as described by Festinger's Social Comparison Theory [6-8]. One study of patients with eating disorders (ED) revealed that the extent of body-related social comparison was strongly correlated with eating disorder symptoms [9], such that comparison of one's own body with the thin ideal was a risk factor for the development of eating disorders.

One body shape comparison study reported that patients with AN exhibit greater activation of the right sensorimotor regions (insula and premotor cortex) and reduced activation of the pregenual ACC during self-other comparisons of body shape compared with healthy controls [10]. However, because idealized body images were the only visual stimuli tested, it is unclear whether participants compared themselves with the models in terms of slimness (i.e. social comparison) or if they were responding only to the "human body" component of the stimuli. This issue could be clarified by presenting a control condition not involving social comparisons by using additional body images, such as images of healthy weight and overweight bodies that would not be expected to trigger negative social comparison. Such an approach may clarify whether anxiety is triggered in patients by the social comparison of their own body image with others' body image. Moreover, the effects of starvation or low body weight on cerebral blood flow in patients with
AN presents another potentially confounding variable. This issue may be ameliorated by testing recovered patients with AN (recAN) [11, 12]. Patients with recAN are considered to have "normal-weight $\mathrm{AN}^{\mathrm{N}}$ in terms of the underlying cognitive pathophysiology and neural function. Patients with recAN are a normal-weight such that they have physiological healthy global cerebral blood flow [13], although they often continue to have modest but persistent dysphoric mood, obsessionality, body image concerns, and body dissatisfaction, as do patients with AN $[14,15]$. However, the cognitive alterations in recAN are less severe than in patients with $\mathrm{AN}$, such that the difference between recAN and AN is a matter of extent [14]. To the best of our knowledge, no previous studies have examined brain activity in patients with recAN during body-related social comparisons. Examining recovered patients may thus provide new insight into the process of recovery from AN.

In the present study, we used functional magnetic resonance imaging (fMRI) to investigate the neural correlates of body comparisons in recAN and control participants. We examined cerebral responses during the comparison between the self and images of others' bodies, with visual stimuli depicting features of underweight, healthy weight, and overweight variants of a canonical female body, to reveal the specific impact of social comparison between the self and underweight female body images on brain responses in patients with recAN.

There are two components that cause body image distortion in AN: 1) perceptual disturbances and 2) dissatisfaction with one's own body as a result of social comparison [16]. Accordingly, we used two experimental tasks to investigate each of the two disturbances. In the first, participants estimated the weight of a female body from a photograph ("weight estimation task"), which draws attention to others' body shapes and weights. In the second, participants compared their own body shape with another female body in a photograph and were asked to rate their subjective anxiety level ("comparison task"). When perceiving images of bodies during the weight estimation task, patients with recAN are hypothesized to show reduced activity in brain systems concerned with processing body size/image than do healthy controls due to abnormalities in the visual processing of body size and shape. However, during the comparison task, we hypothesized that patients with recAN would report more anxiety and display higher activity in emotion-related brain regions. More specifically, we focused on areas that have been reported in previous research in which patients compared their own body with an idealized slim body shape [17]. These regions included the left pregenual ACC, right dorsolateral prefrontal, right inferior parietal lobule, right lateral fusiform gyrus, and left lateral fusiform gyrus [17]. In addition, we performed an exploratory whole-brain analysis to identify group differences. 


\section{Methods}

The study was approved by the local Ethics Committee (National Center of Neurology and Psychiatry) and conducted in accordance with the Declaration of Helsinki.

\section{Participants}

We recruited 15 women who had recovered from AN (recAN), nine of whom recovered from the restricting subtype and six from the binge/purge subtype. We also recruited 14 healthy women who had never suffered from any ED (controls). One patient with recAN was excluded due to movement artifacts in the images, and one was excluded because of the presence of current AN symptoms. Another patient with recAN dropped out because of retraction of consent to participate. Finally, one control participant was excluded due to excessive image distortion within the frontal cortex caused by a hardware error. Consequently, the final analysis included 12 women with recAN (seven restricting and five binge/purge subtype) and 13 control participants. Two patients with recAN who recovered from the restricting subtype were receiving fluvoxamine. One with recAN who recovered from the restricting subtype was receiving sertraline. One with recAN who recovered from the binge/purge subtype was receiving paroxetine and etizolam. One with recAN who recovered from the binge/purge subtype was receiving fluvoxamine, ethyl loflazepate, and etizolam. One patient with recAN who recovered from the restricting subtype met the criteria for generalized anxiety disorder. One patient with recAN who recovered from the binge/purge subtype met the criteria for obsessive compulsive disorder. The two groups were matched in terms of age, education, and predicted IQ (Table 1).

All women with recAN were recruited between February 2011 and August 2013 from Nobino-Kai, a non-governmental self-help support organization specializing in ED in Kanagawa, Japan. All recAN participants underwent two screening phases: (1) a brief phone screening with a clinical psychologist or medical doctor; and (2) a comprehensive assessment using a structured psychiatric interview (the Mini-International Neuropsychiatric Interview [MINI] $[18,19])$ and a face-to-face interview and physical examination with a medical doctor with experience in ED. Control participants were recruited through our healthy participant database and public advertisements. Control participants had no history of an eating disorder or any psychiatric or serious medical or neurological illness, no first-degree relatives with an eating disorder, and had been within normal weight range since menarche.

Inclusion criteria for recAN included having previously met DSM-IV criteria for AN, with subsequent successful recovery from AN. The definition of recovery was no longer meeting the DSM-IV criteria for AN, including maintaining a weight above $85 \%$ of the standard body weight, having regular menstrual cycles, and not having engaged in binge eating, purging, or significant restrictive eating patterns for at least 1 year before the study. None of the participants met the exclusion criteria for alcohol or drug abuse or dependence, major depressive disorder, or severe anxiety disorder within 3 months before the experiment. General exclusion criteria included a history of head injury, hearing or visual impairment, any neurological disease, metallic implants or claustrophobia. Participants were asked to avoid eating and drinking caffeinated beverages for $2 \mathrm{~h}$ and alcohol for $24 \mathrm{~h}$ preceding the experiment.

Participants were informed that they were taking part in a study investigating the neural processing of body-related information. We asked participants who completed the experiment not to tell other participants anything about the experiment. Participants who completed the experiment left the experiment room without meeting other participants.

\section{Preparation of task stimuli}

We employed and took photographs of three female models who were 21 years old and $160 \mathrm{~cm}$ tall, but were within three different body weight categories (underweight: $41 \mathrm{~kg}$, body mass index [BMI] $16 \mathrm{~kg} / \mathrm{m}^{2}$; healthy weight: $54 \mathrm{~kg}$, BMI $21 \mathrm{~kg} / \mathrm{m}^{2}$; and overweight: $75 \mathrm{~kg}$, BMI $29 \mathrm{~kg} / \mathrm{m}^{2}$ [20, 21]. Example photographs are shown in Fig. 1. All photographs were taken in the same room

Table 1 Comparisons of demographic and clinical characteristics between the patient and control groups

\begin{tabular}{|c|c|c|c|c|}
\hline & \multicolumn{2}{|l|}{ Mean \pm standard error } & \multirow[t]{2}{*}{ Student's $t$} & \multirow[t]{2}{*}{$p$-value } \\
\hline & Patients with recAN & Controls & & \\
\hline Age (years) & $33.2 \pm 0.08$ & $29.7 \pm 2.83$ & -1.00 & 0.33 \\
\hline BMI $\left(\mathrm{kg} / \mathrm{m}^{2}\right)$ & $20.7 \pm 0.71$ & $21.5 \pm 0.714$ & 0.80 & 0.43 \\
\hline Education (years) & $14.3 \pm 0.71$ & $14.3 \pm 0.74$ & 1.45 & 0.16 \\
\hline Predicted IQ & $108.1 \pm 2.73$ & $109.1 \pm 2.53$ & -0.27 & 0.78 \\
\hline Lowest past BMI $\left(\mathrm{kg} / \mathrm{m}^{2}\right)$ & $12.9 \pm 0.53$ & - & - & - \\
\hline Age of onset (years) & $17.6 \pm 0.77$ & - & - & - \\
\hline Length of recovery (years) & $5.6 \pm 1.19$ & - & - & - \\
\hline
\end{tabular}

rec $A N$ recovered anorexia nervosa, $B M I$ body mass index 

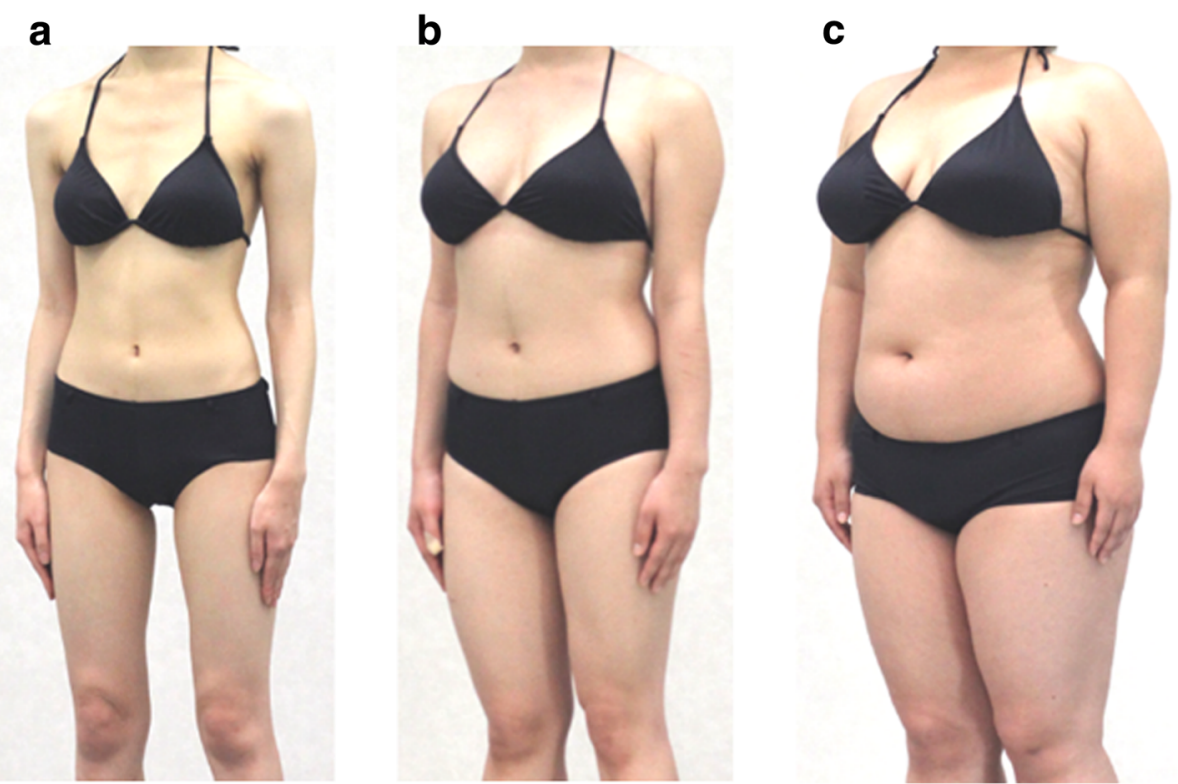

Fig. 1 Examples of body images presented in the task during the fMRI scanning. We presented three types of photographs of female models with different body shapes: (a) underweight (41 kg, body mass index [BMI] $\left.16 \mathrm{~kg} / \mathrm{m}^{2}\right)$; (b) healthy weight $\left(54 \mathrm{~kg}, \mathrm{BMI} 21 \mathrm{~kg} / \mathrm{m}^{2}\right)$; and (c) overweight $\left(75 \mathrm{~kg}, \mathrm{BMl} 29 \mathrm{~kg} / \mathrm{m}^{2}\right)$. The three models were the same age (21 years old) and height $(160 \mathrm{~cm})$

under identical conditions, with the models wearing a uniform black bikini in front of a white wall. Each woman was photographed in two postures (placing the arms behind the head, or down beside the body) from 12 angles (in 30-degree increments) for each posture, from the neck down $[22,23]$. Three sets of 24 photographs (72 photographs in total) of each model's body were used as stimuli.

\section{fMRI parameters}

MR images were acquired using a $1.5 \mathrm{~T}$ Siemens Magnetom Vision Plus System. To obtain functional imaging with a blood-oxygenation-level-dependent (BOLD) contrast, changes in the $\mathrm{T} 2 \%$-weighted MR signal were measured with a gradient echo-planar imaging (EPI) sequence (repetition time $[\mathrm{TR}]=2500 \mathrm{~ms}$, echo time $[\mathrm{TE}]=40 \mathrm{~ms}$, field of view $[\mathrm{FOV}]=192 \mathrm{~mm}$, flip angle $=90$ degree, $64 \times 64$ matrix, 31 slices per slab, slice thickness $=3.5 \mathrm{~mm}, 1 \mathrm{~mm}$ gap along the AC-PC plane). We conducted four functional imaging runs to obtain 227 EPI volume images in each run, with the first five volumes discarded because of instability of magnetization. Thus, the final analysis included four sets of the remaining 222 EPI volumes in each run, resulting in 888 volumes.

\section{Experimental paradigm and procedure}

In each functional imaging run, participants performed repeated event-related trials. In each trial, participants were exposed to one body photograph presented on a screen, followed by either the "comparison task" or the "weight estimation task". In the comparison task, participants were asked to compare their own body shape with the presented body photograph, and to rate their own subjective anxiety level in response to the photograph using four buttons on an MRI-compatible button pad (1 = "calm", 2 = "somewhat calm", 3= "slightly anxious", and $4=$ "anxious"). In the weight estimation task, participants were required to objectively estimate the weight of the body in the photograph, selecting one of four weight categories $(35,55,65$, or $80 \mathrm{~kg})$ using the 4-button pad. Each trial started by indicating the task by displaying "comparison task" or "weight estimation task" for $2.5 \mathrm{~s}$, followed by the task response, lasting for $5 \mathrm{~s}$. Each trial ended with a fixation cross that continued to be presented during a $7.5 \mathrm{~s}$ inter-trial interval. The entire scanning procedure consisted of four separate runs, each containing 36 stimuli in a randomized order (Fig. 2). Instructions and body photographs were presented on a rear-projection screen, viewed via a mirror fitted to the MRI head coil. Response times from the start of the task to the button press were measured in each trial.

To measure anxiety levels more precisely in response to each photo in the comparison task, participants also rated the images after leaving the scanner. Participants were presented with each photo together with the following written question: "How do you feel when comparing your own body shape with this body image?" They responded on a 7 -point scale for anxiety ( $1=$ "very calm", $2=$ "calm", 3 = "somewhat calm", $4=$ "neutral", $5=$ "slightly anxious", 6 $=$ "anxious", or $7=$ "very anxious"). To measure the 


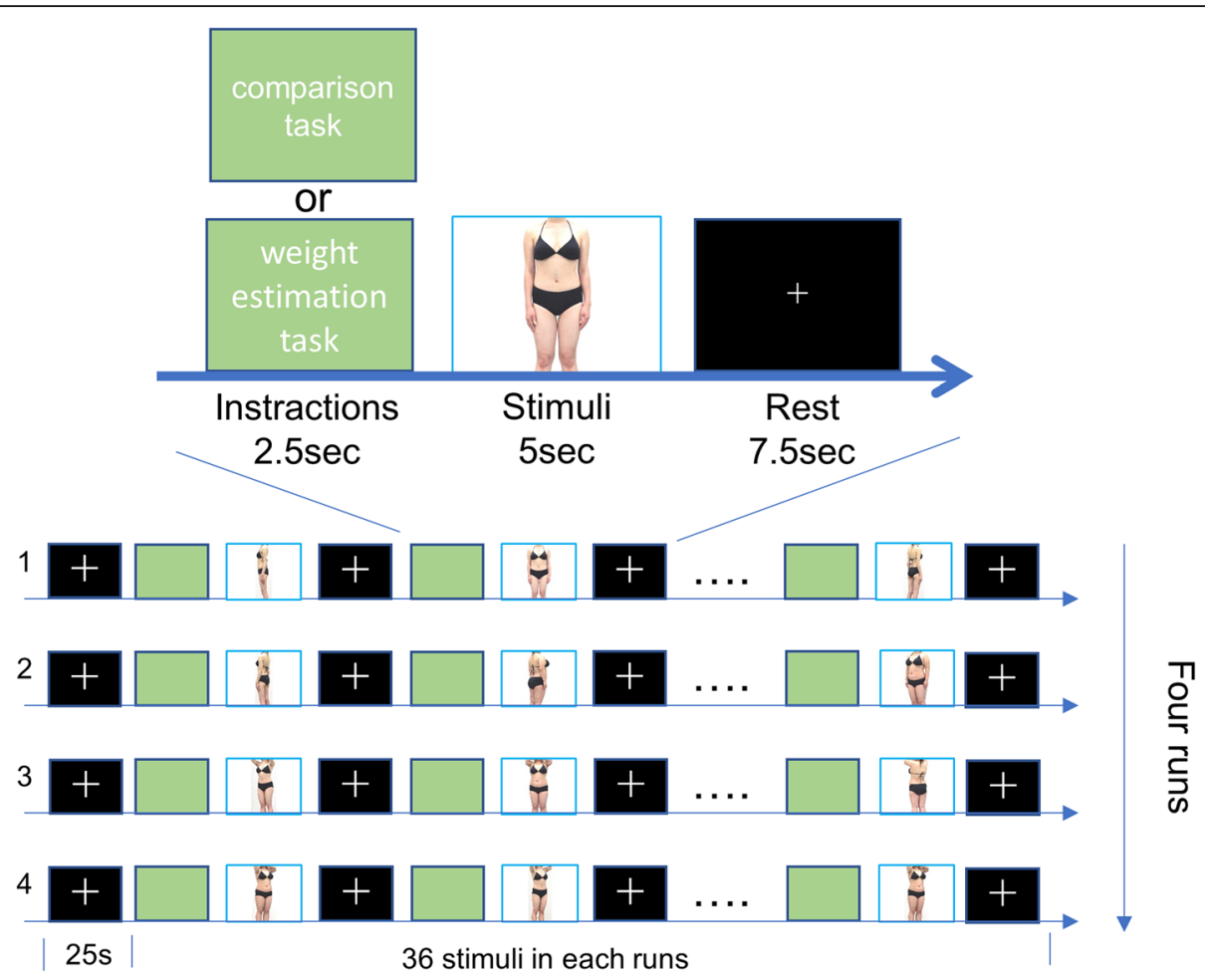

Fig. 2 fMRI paradigm. We conducted four functional imaging runs. In each trial, participants were exposed to one body photograph presented on the screen, followed by either the "comparison task" or the "weight estimation task". In the comparison task, participants were asked to compare their own body shape with the presented body photograph, and to rate their own subjective anxiety level in response to the photograph using four buttons on an MRI-compatible button pad ( $1=$ "calm", 2 = "somewhat calm", 3 = "slightly anxious", and $4=$ "anxious"). In the weight estimation task, participants were required to objectively estimate the weight of the body in the photograph, selecting one of four weight categories $(35,55,65$, or $80 \mathrm{~kg})$ using the 4-button pad. Each trial started by indicating the task with presentation of "comparison task" or "weight estimation task" for $2.5 \mathrm{~s}$, followed by the task response, lasting for $5 \mathrm{~s}$. Each trial ended with a fixation cross that continued to be presented during a $7.5 \mathrm{~s}$ inter-trial interval

accuracy of weight estimation in more detail, participants rated the photos used in the weight estimation task on a visual analogue scale (VAS), with a written question: "How much do you estimate the person's body shown in the image weighs?" The VAS line ranged from 10 to $90 \mathrm{~kg}$, with $10 \mathrm{~kg}$ increments.

Participants completed the following battery of self-report questionnaires: the Eating Disorder Examination Questionnaire (EDE-Q), an established assessment of behavioral and attitudinal eating pathology $[24,25]$; the Eating Disorder Inventory-2 (EDI-2), a validated self-report instrument measuring eating problems [26, 27]; the 21-item Beck Depression Inventory Version 2 (BDI-2), a widely-used measure of psychological and physical symptoms of depression in adults [28, 29]; and the State-Trait Anxiety Inventory (STAI), a validated tool for evaluating anxiety including two dimensions (state anxiety (Y-1), which evaluates the emotional state of an individual in a particular situation, and trait anxiety (Y-2), which refers to a relatively stable personality characteristic $[30,31])$. Finally, we employed the Japanese version of National Adult Reading Test (JART) [32] as a convenient alternative tool to measure participants' IQ.

\section{fMRI data analyses}

Image processing and statistical analyses were conducted with Statistical Parametric Mapping, version 12 (SPM12, Wellcome Department, London, UK https://www.fil.ion.ucl.ac.uk/spm/software/spm12/). Preprocessing of functional scans included realignment of functional images for motion correction using the first scan as a reference and spatial normalization to a standard template (Montreal Neurological Institute; MNI) with a resampled voxel size of $2 \times$ $2 \times 2 \mathrm{~mm}$. Spatial smoothing with an $8 \mathrm{~mm}$ Gaussian kernel was applied to the analyzed images.

For first-level within-participant analysis, a design matrix for an individual general linear model (GLM) was constructed, incorporating six regressors corresponding to the 2 task types (comparison or weight estimation) $\times$ 3 types of weight (underweight, healthy weight, or overweight). The regressors were hypothetical hemodynamic responses modeled with the onset of corresponding events and convolved with a canonical hemodynamic 
response function. Intrinsic autocorrelations were accounted for by $\mathrm{AR}(1)$ and low frequency drift was removed via a high pass filter (128 s) in individual GLM analyses. The six 3D beta images including beta estimates from the GLM were fed into the subsequent second-level group analysis.

At the second level, a group analysis was performed using a full factorial design with one between-participants and one within-participants factor for testing the 2-way group (recAN or controls) $\times$ weight (underweight, healthy weight, or overweight) interaction effect during each of the comparison and weight-estimation tasks.

Our primary statistical analyses investigated weightby-group interactions in constrained regions of interest (ROIs) during each of the comparison and weight-estimation tasks. We localized ROIs in the ACC, dorsolateral prefrontal, inferior parietal lobules, and lateral fusiform gyrus (FG), based on activations reported in a previous study of a body comparison task [17]. Each ROI was defined by a $12 \mathrm{~mm}$ radius sphere centered on the reported Montreal Neurological Institute (MNI) coordinates (left pregenual ACC: $x=-7, y=34, z=5$; right dorsolateral prefrontal: $x=47, y=2, z=36$; right inferior parietal lobule: $x=52$, $y=-33, z=42$; right lateral fusiform gyrus: $x=x=47, y=$ $-57, z=-15$; left lateral fusiform gyrus: $x=-47, y=-65$, $z=-12$ ). We created a single template for all ROIs using the MarsBaR version 0.44 (available at http://marsbar.sourceforge.net/) [33]. The small volume correction method was used to investigate the ROIs as a whole. We also performed voxel-by-voxel analyses within a whole set of ROIs, with the SVC method and the statistical significance level set at $p<0.05$ corrected for family-wise error (FWE).

To investigate explanatory activation outside predetermined ROIs, we performed an exploratory whole-brain analysis to identify group differences, with a height threshold of $p<0.001$ (uncorrected) and an extent threshold of $p<0.05$ corrected for false discovery rate (FDR) [34].

\section{Statistical analysis}

We compared continuous variables with Student's $t$-tests. When necessary, $t$-tests were modified for unequal variances. For the variables from self-report questionnaires, we used Mann-Whitney $U$ tests. Response times were analyzed with two-way repeated-measures ANOVAs (weight $\times$ group). Statistical analyses of non-imaging data were performed using IBM SPSS Statistics version 24 for Windows (IBM Corp., Chicago, USA), with statistical significance set at $p<0.05$. We used Bonferroni corrections for the multiple comparisons analyses, such as the separate analyses for each stimulus weight.

\section{Results}

\section{Baseline characteristics}

There were no group differences in demographic variables (Table 1). Patients with recAN scored marginally higher on state anxiety (Y-1) than did controls, but the difference was not statistically significant. Patients with recAN scored significantly lower on body dissatisfaction in the EDI-2 and marginally lower on the drive for thinness in the EDI-2 compared with controls. Patients with recAN scored significantly higher on perfectionism than control participants in the EDI-2 (Table 2).

These results indicated that patients with recAN no longer exhibited typical diagnostic symptomatology of AN, but still displayed significantly heightened perfectionism compared with the general population, even after longterm weight restoration and recovery $[17,35]$. Interestingly, reported body dissatisfaction was lower in the recAN group, indicating that recovered patients were, at least subjectively, satisfied with their own body image when not involved in a social comparison situation.

\section{Behavioral results during the task}

There were no significant group differences observed in overall response time $(F(1,23)=0.447, p=0.510$, and $F(1$, 23) $=0.294, p=0.593$ for the comparison and weight estimation tasks, respectively). We also did not observe any significant group differences in anxiety levels on the 4-point scale during the comparison task in the scanner $(F(1,23)=$ $0.41, p=0.84)$, and this result was confirmed on the 7-point scale for anxiety after scanning $(F(1,23)=0.13, p=0.725)$.

Compared with controls, patients with recAN marginally underestimated the weight of female body photos during scanning, but the difference did not reach statistical significance $(F(1,23)=4.20, p=0.052$; Table 3$)$. These results were also reflected by the VAS scale estimations after scanning $(F(1,23)=0.001, p=0.997)$. However, more detailed analysis revealed that patients with recAN underestimated the weights of underweight body photos (approximately $41 \mathrm{~kg}$ in reality) compared with controls. This was indicated by a significant weight $\times$ group interaction on body weight estimation $(F(2,46)$ $=8.02, p=0.001$; two-way repeated measures ANOVA), and by lower weight estimation values in recAN than in controls (recAN: mean $\pm \mathrm{SE}=39.2 \pm 1.21 \mathrm{~kg}$; controls: $42.5 \pm 1.00 \mathrm{~kg}, p=0.04$, Bonferroni-corrected). Controls tended to underestimate the weight of overweight female body images (representing $75 \mathrm{~kg}$ ) more than patients with recAN (recAN: mean $\pm \mathrm{SE}=67.7 \pm 1.50 \mathrm{~kg}$; controls: $64.3 \pm 1.15 \mathrm{~kg}, p=0.08$; Table 4).

In summary, the two groups showed similar subjectively reported anxiety levels during self-other body shape comparison. However, estimation of other's body weight differed between recAN patients and controls. There were no differences in behavioral 
Table 2 Questionnaires characterizing participants' inner body image and affect

\begin{tabular}{|c|c|c|c|c|}
\hline & Mean \pm standard erro & & Mann-Whitney U & $p$-value \\
\hline & Patients with recAN & Controls & & \\
\hline EDE-Q score & & & & \\
\hline Restraint & $0.2 \pm 0.12$ & $0.4 \pm 0.18$ & 56.0 & 0.38 \\
\hline Eating concern & $0.6 \pm 0.22$ & $0.4 \pm 0.14$ & 65.0 & 0.71 \\
\hline Weight concern & $1.4 \pm 0.34$ & $1.4 \pm 0.34$ & 66.5 & 0.76 \\
\hline Shape concern & $1.5 \pm 0.34$ & $1.7 \pm 0.32$ & 66.0 & 0.76 \\
\hline EDI-2 score & & & & \\
\hline Drive for thinness & $1.9 \pm 0.74$ & $4.7 \pm 1.12$ & 42.5 & $0.052+$ \\
\hline Bulimia & $2.1 \pm 1.32$ & $0.92 \pm 0.47$ & 74.0 & 0.85 \\
\hline Body dissatisfaction & $5.5 \pm 1.65$ & $12.0 \pm 1.94$ & 35.5 & $0.02^{*}$ \\
\hline Ineffectiveness & $9.0 \pm 2.10$ & $4.5 \pm 0.89$ & 48.5 & 0.11 \\
\hline Perfectionism & $5.7 \pm 0.95$ & $1.9 \pm 0.70$ & 20.0 & $0.001^{* *}$ \\
\hline Interpersonal distrust & $4.9 \pm 1.18$ & $2.5 \pm 0.77$ & 48.0 & 0.11 \\
\hline Interoceptive awareness & $3.7 \pm 1.79$ & $2.2 \pm 0.52$ & 76.0 & 0.94 \\
\hline Maturity fears & $3.3 \pm 0.87$ & $4.6 \pm 0.95$ & 56.0 & 0.25 \\
\hline BDI-II & $11.0 \pm 3.64$ & $11.0 \pm 3.40$ & 73.5 & 0.81 \\
\hline STAI(Y-1) & $45.0 \pm 3.36$ & $36.8 \pm 2.23$ & 44.5 & $0.07 \dagger$ \\
\hline STAI(Y-2) & $46.7 \pm 3.13$ & $42.6 \pm 2.50$ & 63.5 & 0.44 \\
\hline
\end{tabular}

$+p<0.1 * p<0.05 * * p<0.01$

EDI-Q Eating Disorder Examination Questionnaire, EDI-2 Eating Disorder Inventory-2, BDI-II Beck Depression Inventory Version 2, STAI(Y-1) State-Trait Anxiety Inventory, state anxiety, STAI(Y-2) State-Trait Inventory, trait anxiety

results between the patients with past restricting AN and those with past binge/purging AN.

\section{fMRI results}

\section{ROI analyses}

We observed a significant weight $\times$ group interaction effect during the comparison task in the left pregenual ACC (peak cluster coordinate: $x=-10, y=36, z=-2$; cluster size: $k=6$ voxels; $F(2,138)=13.87, p=0.008$, FWE; Fig. 3,

Table 3 Rating scores for the comparison task and weight estimation task

\begin{tabular}{|c|c|c|c|c|}
\hline & \multicolumn{2}{|c|}{ Mean \pm standard error } & \multirow[t]{2}{*}{$F$ value $(1,23)$} & \multirow[t]{2}{*}{$p$-value } \\
\hline & $\begin{array}{l}\text { Patients with } \\
\text { recAN }\end{array}$ & Controls & & \\
\hline \multicolumn{3}{|l|}{ Comparison task } & 0.41438 & 0.84 \\
\hline Underweight & $2.1 \pm 0.26$ & $1.6 \pm 0.22$ & & 0.22 \\
\hline Healthy weight & $2.1 \pm 0.19$ & $2.0 \pm 0.20$ & & 0.82 \\
\hline Overweight & $3.3 \pm 0.07$ & $3.4 \pm 0.09$ & & 0.11 \\
\hline \multicolumn{3}{|c|}{ Weight estimation task } & 4.2022 & $0.052 \dagger$ \\
\hline Underweight & $1.1 \pm 0.08$ & $1.3 \pm 0.10$ & & 0.12 \\
\hline Healthy weight & $2.0 \pm 0.02$ & $2.2 \pm 0.04$ & & $0.03^{*}$ \\
\hline Overweight & $3.3 \pm 0.07$ & $3.4 \pm 0.09$ & & 0.25 \\
\hline
\end{tabular}

Additional file 1: Tables S1-S3) such that patients with recAN showed greater pregenual ACC activation in response to underweight images compared with controls ( $T=3.47, p=0.002$, thresholded by Bonferroni correction).

In contrast, we did not observe a weight $\times$ group interaction effect during the weight estimation task on activation in any ROI.

Table 4 7-point scale for anxiety and VAS scores for weight estimation

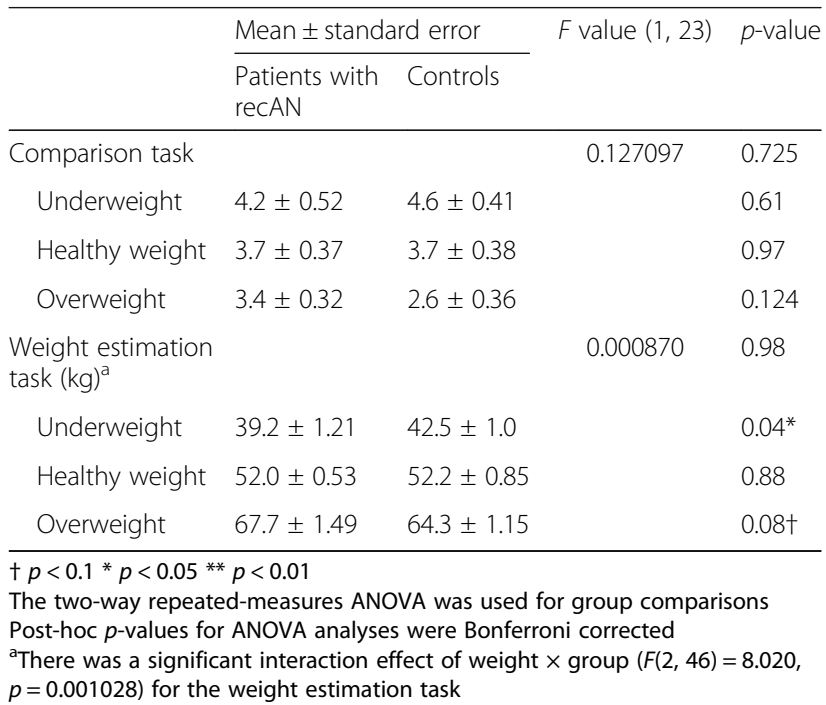




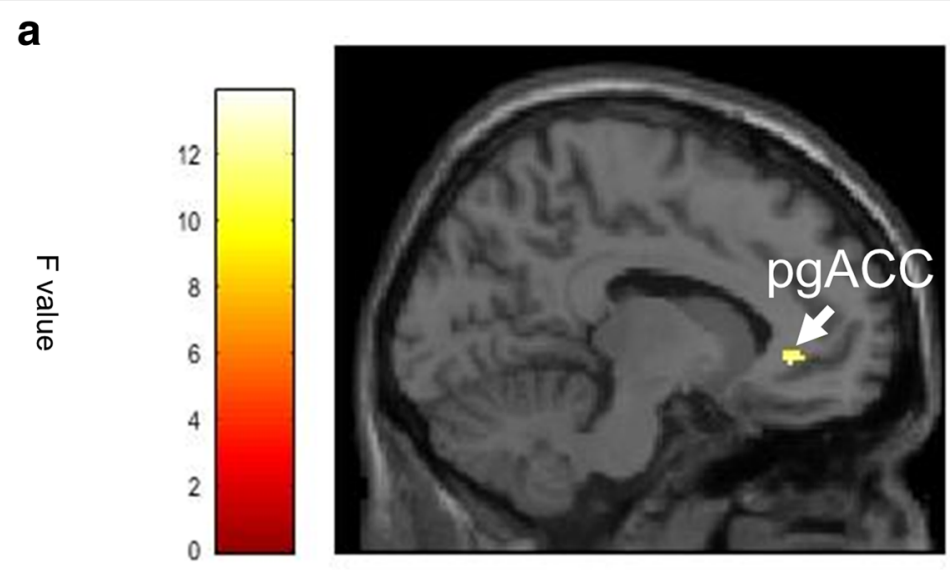

b

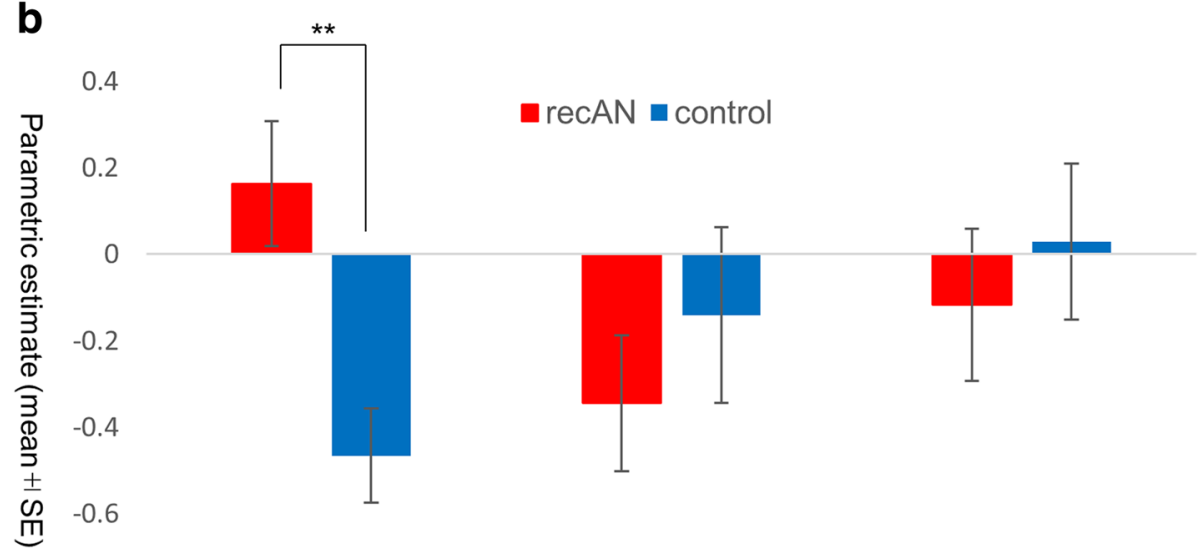

$-0.8$

\section{Underweight Healthy weight Overweight}

Fig. 3 Group-by-stimulus 2-way interactive effect on BOLD signals during the comparison task. a The cluster rendered on the sagittal brain section shows the group-by-stimulus 2-way ANOVA interactive effect for the comparison task on BOLD signals in the left pregenual anterior cingulate (pgACC; the maximum peak of the effect at the MNI coordinates: $x=-10, y=36, z=-2 \mathrm{~mm}$; cluster size: $k=6$ voxels; peak $Z$ score $=4.30, p=0.008$, FWE corrected for multiple comparisons). The analysis was based on a region of interest (ROI) analysis, with small volume correction (SVC) within the left pregenual ACC (a 12-mm radius sphere centered on $x=-7, y=34, z=5$ ). The left color bar indicates the $F$ value of the rendered cluster. $\mathbf{b}$ The bar graph shows the parametric estimates from the ANOVA analysis of the BOLD signals in the left pgACC cluster in each group (recAN and control) for each stimulus type (underweight, healthy weight, and overweight). Post hoc $t$-tests showed significantly greater BOLD signals in patients with recAN than in controls. Error bars indicate standard error. ${ }^{*} p<0.05$ (Bonferroni-corrected), ${ }^{* *} p<0.01$ (Bonferroni-corrected).. MNI: Montreal Neurological Institute; BOLD: blood oxygen level dependent; FWE: family-wise error; recAN: recovered anorexia nervosa

\section{Whole brain analysis}

In addition, we explored group differences in whole brain responses to all body images (irrespective of weight) during the comparison and weight-estimation tasks. The patients with recAN exhibited greater visual cortex activation than did control participants during the comparison task (left superior occipital gyrus [BA17]; peak coordinate $x=-6, y=-$ 106, $z=4, T=5.07$, peak level $p=0.0000016$ (uncorrected), cluster level $p=0.033$ (FDR); Fig. 4, Additional file 1: Tables S1-S3). These results suggest that patients with recAN exhibit heightened attention to other's body shapes, irrespective of weight during self-other comparisons of body shape. We also conducted post-hoc Bonferroni corrected $\mathrm{T}$ tests to examine the difference between those with a history of restricting and binge/purging AN. Patients with recAN who recovered from restricting $\mathrm{AN}$ (recANr) exhibited greater visual cortex activation than did patients with recAN who recovered from binge/purging $\mathrm{AN}$ (recANbp) (parametric estimates recANr: mean $\pm \mathrm{SE}=6.77 \pm 0.90$, recANbp: mean $\pm \mathrm{SE}=4.29 \pm 1.07 T=2.94, p=0.008$ ). When we analyzed separately for each weight group (underweight, healthy weight, or overweight), there was no significant difference between those with a history of restricting $\mathrm{AN}$ and those with a history of binge/purging AN.

In contrast, patients with recAN exhibited weaker activation during weight estimation in the middle temporal gyrus ([BA37]; peak coordinate $x=48, y=-58, z=-2$, $T=4.49$, peak level $p=0.0000139$ (uncorrected), cluster 

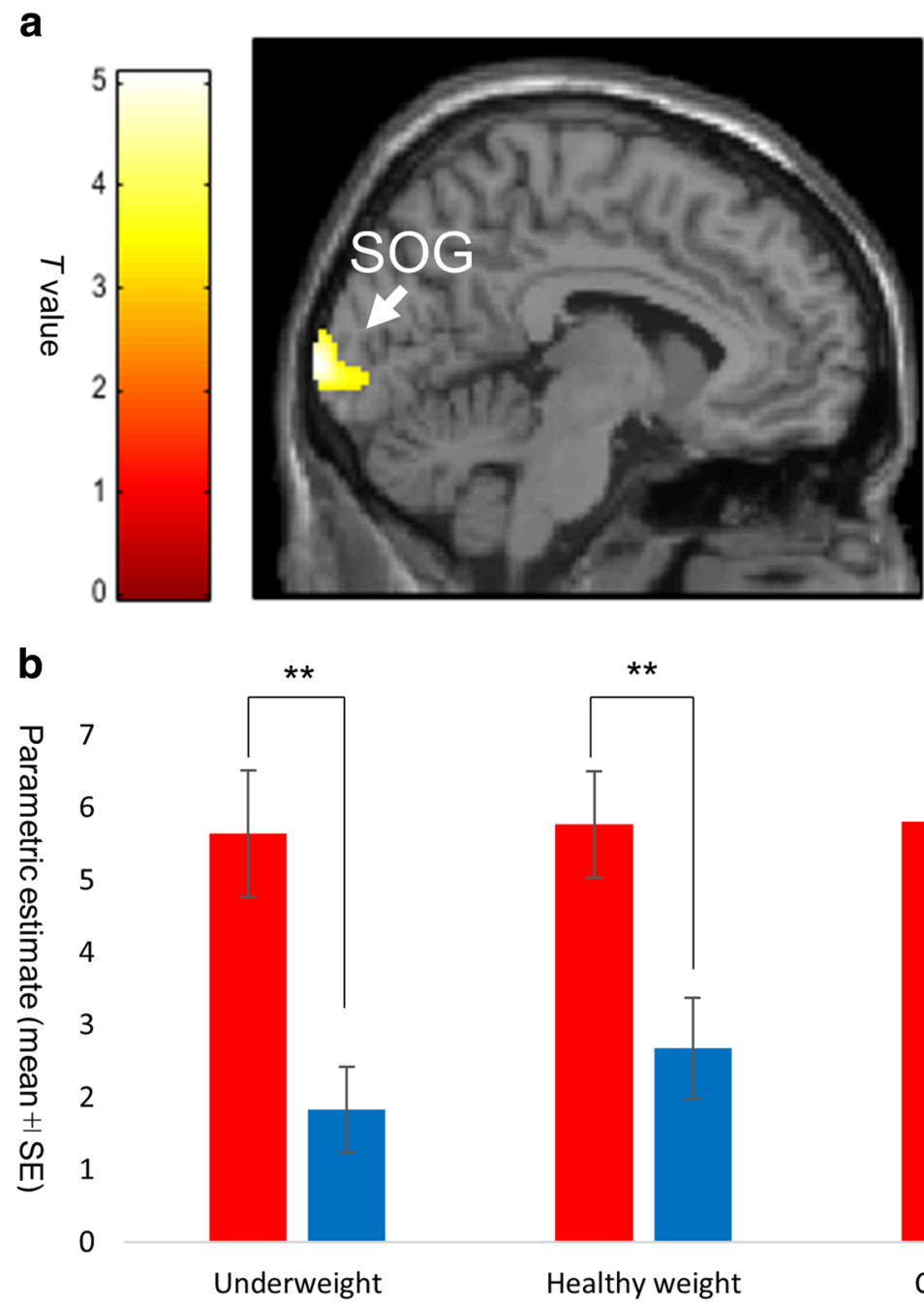

\section{Healthy weight \\ recAN control}

Fig. 4 Group comparison (recAN > controls) of the brain BOLD signals during the comparison task. a The cluster rendered on the sagittal brain section shows the group effect (recAN > controls) in the comparison task on BOLD signals in the left visual cortex (left superior occipital gyrus). Maximum peak of the effect at the MNI coordinates: $x=-6, y=-106, z=4 \mathrm{~mm}$; cluster size: $k=362$ voxels; peak $T$ score $=5.07$, height level: $p=$ $1.6 \times 10^{-6}$ (uncorrected), cluster level: $p=0.033$; FDR corrected for multiple comparisons. The analyses were on a whole brain exploratory voxelby-voxel basis. The left color bar indicates the $T$ value of the rendered cluster. $\mathbf{b}$ The bar graph shows the parametric estimates of the BOLD signals in the left SOG cluster in each group (recAN and controls) for each stimulus type (underweight, healthy weight, and overweight). Post hoc $t$-tests showed significantly greater BOLD signals in patients with recAN than in controls, regardless of the stimulus types. Error bars indicate standard error. ${ }^{*} p<0.05,{ }^{* *} p<0.01$ (Bonferroni-corrected). MNI: Montreal Neurological Institute; BOLD: blood oxygen level dependent; FDR: false discovery rate; $S O G$, superior occipital gyrus; recAN: recovered anorexia nervosa

level $p=0.029$ (FDR), corresponding to the extrastriate body area, EBA [36]; Fig. 5, Additonal file 1: Table S4). This suggests that patients with recAN exhibited altered visual processing specific to human bodies.

\section{Discussion}

In the current study, we investigated the neural underpinnings of body shape comparisons and weight estimation in patients with recAN. Our fMRI results identified differential neural correlates of self-other comparisons of body shape between patients with recAN and controls.
Our hypothesis was supported by the result that patients with recAN showed reduced activation of the right EBA during the weight estimation task. A potential advantage of our study is that patients with recAN of a healthy weight were not affected by structural cortical volume loss, which is evident in women suffering from ongoing AN, in the left lateral occipital cortex including the EBA [37]. Nevertheless, the current results seem consistent with a previous report that women with ongoing AN exhibited reduced EBA activation [38] and reduced effective connectivity with the fusiform body area 


\section{a}
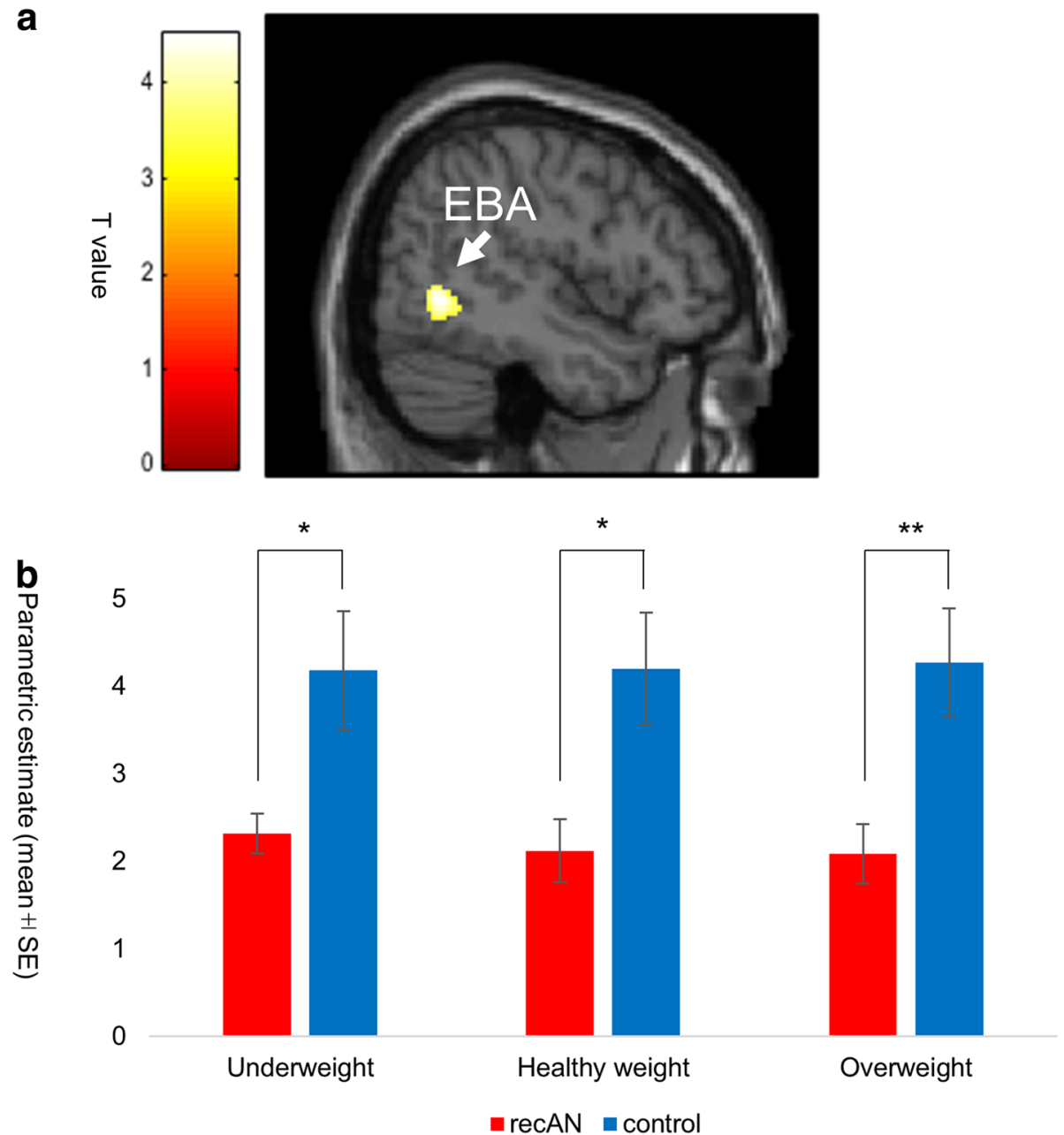

Fig. 5 Group comparison (controls > recAN) of the brain BOLD signals during the weight estimation task. a The cluster rendered on the sagittal brain section shows the group effect (control > recAN) in the weight estimation task on BOLD signals in the middle temporal gyrus corresponding to the extrastriate body area (EBA, Brodmann's area 37). The maximum peak of the effect at the MNI coordinates: $x=48, y=-58, z=-2 \mathrm{~mm}$; cluster size: $k=$ 131 voxels; peak $T$ score $=4.49$, height level: $p=1.39 \times 10^{-5}$ (uncorrected), cluster level: $p=0.029$; FDR corrected for multiple comparisons. The analyses were on a whole brain exploratory voxel-by-voxel basis. The left color bar indicates the $T$ value of the rendered cluster. $\mathbf{b}$ The bar graph shows the parametric estimates of the BOLD signals in the right EBA cluster in each group (recAN and controls) for each stimulus type (underweight, healthy weight, and overweight). Post hoc t-tests showed significantly greater BOLD signals in controls than in patients with recAN, regardless of the stimulus types. Error bars indicate standard error. ${ }^{*} p<0.05,{ }^{*} p<0.01$ (Bonferroni-corrected). MNI: Montreal Neurological Institute; BOLD: blood oxygen level dependent; FDR: false discovery rate; recAN: recovered anorexia nervosa

[39]. The EBA does not respond to objects or parts of objects, but to human bodies and body parts such as hands and feet $[36,40,41]$. The decrease in EBA activity in patients with recAN suggests that altered neural processing of body information persists in patients who have had AN even after long-term recovery from the disorder (approximately 5-6 years). This may underlie the altered perception of others' body shapes, and may be related to the high relapse rate (approximately 35\%) in patients with recAN $[42,43]$.

Contrary to our hypothesis, there was no difference in anxiety levels during body shape comparisons. However, we observed that patients with recAN exhibited greater pregenual ACC activation than did controls during comparisons of their own body with images of an underweight body. The pregenual ACC is involved in detecting conflict in the emotional domain and recruiting cognitive control processes to resolve such conflict [44] [45]. A study of a similar self-other body-shape comparison task reported that healthy women exhibited activation in the left ACC [17], while patients with current AN exhibited reduced activation in the pregenual ACC [10]. A possible reason for the difference between our finding and those from previous reports is that recovery from AN may involve the recovery of top-down control of negative emotional impact during 
self-other comparison of body images, which may cause compensatory hyperfunction in the pregenual ACC.

At baseline, we observed that body dissatisfaction was lower among patients with recAN than among control participants, while anxiety levels during the self-other body comparison task did not differ between the groups. The significantly lower level of body dissatisfaction in recAN compared with controls appears to differ from findings reported in previous studies [35] [46]. However, this finding might be in accord with previous reports that weight regain is accompanied by significant reductions in body dissatisfaction. In our study, body dissatisfaction in recAN was even lower than that of healthy controls, although body dissatisfaction of recAN has been reported to be comparable to that of healthy controls [47]. This might partially be due to cultural background in a Japanese sample, such as the presence of higher body dissatisfaction in Japanese healthy women than in women from other countries (e.g., [48]). Such cultural differences may result in inconsistencies between findings from Japan and Western countries. Nonetheless, this is a matter of speculation and remains to be clarified. We observed that patients with recAN exhibited heightened perfectionism, consistent with previous reports that perfectionism persists among patients with AN who have achieved long-term weight restoration [35, 49-51] and was a risk factor for recurrence [52, 53]. Our results confirm the persistence of heightened perfectionism in patients with recAN.

During the comparison task, patients with recAN showed greater visual cortex activation, which was likely due to heightened attention in these women compared with controls. It is well established that sustained spatial attention modulates cortical responses in V1 [54, 55]. Heightened brain responses may be associated with exaggerated visual processing of body shapes in AN, given that one of the core symptoms of ED is obsessive preoccupation with body weight and shape. Moreover, patients with recANr showed greater activation of the visual cortex than did those with recANbp. This is consistent with a previous study reporting that patients with restricting AN had a larger attentional bias to threat word stimuli related to ED (e.g., FAT) than did healthy controls, whereas patients with binge-purging AN showed avoidance of threat word stimuli related to ED [56]. Our result suggests that differential patterns of attention allocation between patients with restricting $\mathrm{AN}$ and those with binge purging AN may remain even after recovery.

Several limitations should be considered when interpreting our results. The small number of participants is an obvious limitation, which should be addressed in future studies with larger sample sizes. In addition, the current study only measured subjective ratings, without simultaneous objective measures of arousal or anxiety, such as galvanic skin responses or papillary responses representing autonomic nervous activity. Thus, participants may have become habituated with repeated exposure to similar body image stimuli, potentially reducing signal intensity, as reported in previous imaging studies using fear and threat cues $[57,58]$. Our discussion of $\mathrm{AN}$ is based on the assumption that there are certain similarities between recAN and AN. This may be a limitation of the study because recAN is not completely the same as AN. However, despite the differences between recAN and AN (mostly in physiological and nutrition status) the two have many similarities, especially in terms of psychological disturbances, which are likely persistent across the lifespan. For example, patients with AN show personality traits characteristic of AN (such as obsessive-compulsive personality) even before the onset of illness [52, 53], suggesting that such traits may derive from underlying genetic vulnerabilities. Furthermore, previous studies have reported that core temperament and personality traits persist after recovery from AN [35, 59], possibly due to the genetic background of those with the condition. Although we should be aware that recAN is not completely the same as AN, we believe that we can still discuss disturbances of psychological traits in AN based on the similarities between the two. Nonetheless, similar studies with patients with ongoing AN are warranted in the future. Finally, including a patient sample with current AN would enable direct comparison between patients with $\mathrm{AN}$ and recAN. Future studies comparing patients with $\mathrm{AN}$ and recAN may provide further insight into the recovery process.

\section{Conclusions}

The current study revealed that patients with recAN exhibited greater pregenual ACC activation than did controls during comparisons of their own body with underweight female body images. These findings suggest that recovery from AN may involve the regulation of negative affect in response to body images via the pregenual ACC when a patient compares their own body with idealized underweight body images. In addition, there was reduced right EBA activity in patients with recAN, indicating that altered body image processing in the brain can persist even after recovery from AN.

\section{Additional file}

Additional file 1: Table S1. Correlations (Spearman's rho) between subjective anxiety ratings (7-point scale) and neural activation in response to the comparison task. Table S2. Correlations (Spearman's rho) between body dissatisfaction assessed with EDI-2 scores and neural activation in response to the comparison task. Table S3. Correlations (Spearman's rho) between perfection assessed with EDI-2 scores and neural activation in response to the comparison task. Table S4. Correlations (Spearman's rho) between body dissatisfaction assessed with EDI-2 scores and neural 
activation in response to the weight estimation task. Table S5. Correlations (Spearman's rho) between perfection assessed with EDI-2 scores and neural activation in response to the weight estimation task. (DOCX $25 \mathrm{~kb}$ )

\section{Acknowledgements}

The authors acknowledge the co-operation and contribution of the staff and the women who participated in this study.

\section{Funding}

This work was supported by JSPS KAKENHI Grant Number 23659390.

\section{Availability of data and materials}

Data sharing is not applicable.

\section{Authors' contributions}

NK, YM, MM, TA, HK, MG and GK designed the research. NK, AT and MM collected the data. NK, YM and HA analyzed the data and drafted the manuscript. All authors have approved the final manuscript.

\section{Ethics approval and consent to participate}

This study was approved by the Ethics Committee of the National Center of Neurology and Psychiatry, Tokyo, Japan. All participants provided informed consent. We managed the data to ensure that the participants were not identified.

\section{Consent for publication}

Not applicable.

\section{Competing interests}

The authors declare that they have no competing interests.

\section{Publisher's Note}

Springer Nature remains neutral with regard to jurisdictional claims in published maps and institutional affiliations.

\section{Author details}

${ }^{1}$ Division of Psychosomatic Medicine, Department of Neurology, University of Occupational and Environmental Health, Kitakyushu 807-8555, Japan. ${ }^{2}$ Department of Psychophysiology, National Institute of Mental Health, National Center of Neurology and Psychiatry, Tokyo 187-8553, Japan. ${ }^{3} \mathrm{NPO}$ Corporation Nobinokai, Yokohama 236-0014, Japan. ${ }^{4}$ College of Art and Design, Joshibi University of Art and Design, Sagamihara 252-8538, Japan. ${ }^{5}$ Department of Psychosomatic Research, National Institute of Mental Health, National Center of Neurology and Psychiatry, Tokyo 187-8553, Japan. ${ }^{6}$ Department of Psychosomatic Medicine, Center Hospital of the National Center for Global Health and Medicine, Tokyo 162-8655, Japan. ${ }^{7}$ Department of Psychosomatic Medicine, Graduate School of Medical Sciences, Kyushu University, Fukuoka 812-8582, Japan. ${ }^{8}$ Department of Neurology, University of Occupational and Environmental Health, Kitakyushu 807-8555, Japan. ${ }^{9}$ School of Health Sciences Fukuoka, International University of Health and Welfare, Fukuoka 831-8501, Japan.

Received: 19 May 2018 Accepted: 14 October 2018

Published online: 31 October 2018

\section{References}

1. Jacobi $\mathrm{C}$, et al. Coming to terms with risk factors for eating disorders: application of risk terminology and suggestions for a general taxonomy. Psychol Bull. 2004;130(1):19-65.

2. Stice E. Risk and maintenance factors for eating pathology: a meta-analytic review. Psychol Bull. 2002;128(5):825-48.

3. Stice E, Shaw HE. Role of body dissatisfaction in the onset and maintenance of eating pathology: a synthesis of research findings. J Psychosom Res. 2002;53(5):985-93.

4. Groesz LM, Levine MP, Murnen SK. The effect of experimental presentation of thin media images on body satisfaction: a meta-analytic review. Int J Eat Disord. 2002;31(1):1-16.

5. Thompson JK, et al. Exacting beauty: theory, assessment, and treatment of body image disturbance. Washington, DC: American Psychological Association; 1999.
6. Brown A, Dittmar H. Think "thin" and feel bad: the role of appearance schema activation, attention level, and thin-ideal internalization for young women's responses to ultra-thin media ideals. J Soc Clin Psychol. 2005; 24(8):1088-113.

7. Grabe S, Ward LM, Hyde JS. The role of the media in body image concerns among women: a meta-analysis of experimental and correlational studies. Psychol Bull. 2008;134(3):460-76.

8. Festinger L. A theory of social comparison processes. Hum Relat. 1954;7(2): 117-40.

9. Hamel $A E$, et al. Body-related social comparison and disordered eating among adolescent females with an eating disorder, depressive disorder, and healthy controls. Nutrients. 2012;4(9):1260-72.

10. Friederich $\mathrm{HC}$, et al. Neural correlates of body dissatisfaction in anorexia nervosa. Neuropsychologia. 2010;48(10):2878-85

11. Strigo $\mid A$, et al. Altered insula activation during pain anticipation in individuals recovered from anorexia nervosa: evidence of interoceptive dysregulation. Int J Eat Disord. 2013;46(1):23-33.

12. Wagner $A$, et al. Altered reward processing in women recovered from anorexia nervosa. Am J Psychiatry. 2007;164(12):1842-9.

13. Sheng $M$, et al. Cerebral perfusion differences in women currently with and recovered from anorexia nervosa. Psychiatry Res. 2015;232(2):175-83.

14. Wagner A, et al. Personality traits after recovery from eating disorders: do subtypes differ? Int J Eat Disord. 2006;39(4):276-84.

15. Frank GK, et al. Alterations in brain structures related to taste reward circuitry in ill and recovered anorexia nervosa and in bulimia nervosa. Am J Psychiatry. 2013;170(10):1152-60.

16. Rosen JC, et al. Development of a body image avoidance questionnaire Psychol Assess. 1991;3(1):32.

17. Friederich $\mathrm{HC}$, et al. I'm not as slim as that girl: neural bases of body shape self-comparison to media images. Neuroimage. 2007;37(2):674-81.

18. Sheehan, D.V., et al. The Mini-International Neuropsychiatric Interview (M.I.N I.): the development and validation of a structured diagnostic psychiatric interview for DSM-IV and ICD-10. J Clin Psychiatry. 1998;59 Suppl 20:22-33.

19. Otsubo $T$, et al. Reliability and validity of japanese version of the mini-international neuropsychiatric interview. Psychiatry Clin Neurosci. 2005;59(5):517-26.

20. Fladung $A K$, et al. A neural signature of anorexia nervosa in the ventral striatal reward system. Am J Psychiatry. 2010;167(2):206-12.

21. Pruis TA, Keel PK, Janowsky JS. Recovery from anorexia nervosa includes neural compensation for negative body image. Int J Eat Disord. 2012;45(8): 919-31.

22. Morris JP, Pelphrey KA, McCarthy G. Occipitotemporal activation evoked by the perception of human bodies is modulated by the presence or absence of the face. Neuropsychologia. 2006;44(10):1919-27.

23. Vocks $S$, et al. Neural correlates of viewing photographs of one's own body and another woman's body in anorexia and bulimia nervosa: an fMRI study. J Psychiatry Neurosci. 2010;35(3):163-76.

24. Fairburn CG, Beglin SJ. Assessment of eating disorders: interview or selfreport questionnaire? Int J Eat Disord. 1994;16(4):363-70.

25. Mitsui T, Yoshida T, Komaki G. Psychometric properties of the eating disorder examination-questionnaire in Japanese adolescents. Biopsychosoc Med. 2017:11:9.

26. Garner DM. Eating Disorder Inventory-2 (EDI-2). Odessa, Florida: Psychological Assessment Resources; 1991.

27. Ando T, et al. Variations in the preproghrelin gene correlate with higher body mass index, fat mass, and body dissatisfaction in young Japanese women. Am J Clin Nutr. 2007;86(1):25-32.

28. Beck A, et al. Manual for Beck Depression Inventory-Second Edition (Japanese edition). Tokyo: Nihon Bunka Kagakusha; 2003.

29. Kojima $M$, et al. Cross-cultural validation of the Beck depression inventory-II in Japan. Psychiatry Res. 2002;110(3):291-9.

30. Spielberger CD, Gorsuch R, Lushene RE. Manual for the State Trait Anxiety Inventory. Mountain View, California: Counsulting Psychologist Press; 1983.

31. Katsuharu N, Tadanobu M. Development and validation of Japanese version of state-trait anxiety inventory : a study with female subjects. Jpn J Psycho Med. 1982;22(2):107-12.

32. Matsuoka $\mathrm{K}$, et al. Estimation of premorbid IQ in individuals with Alzheimer's disease using Japanese ideographic script (kanji) compound words: Japanese version of National Adult Reading Test. Psychiatry Clin Neurosci. 2006;60(3):332-9.

33. Brett $M$, et al. Region of interest analysis using an SPM toolbox. Sendai: 8th international conference on functional mapping of the human brain; 2002. 
34. Woo CW, Krishnan A, Wager TD. Cluster-extent based thresholding in fMR analyses: pitfalls and recommendations. Neuroimage. 2014;91:412-9.

35. Srinivasagam NM, et al. Persistent perfectionism, symmetry, and exactness after long-term recovery from anorexia nervosa. Am J Psychiatry. 1995; 152(11):1630-4.

36. Downing PE, et al. A cortical area selective for visual processing of the human body. Science. 2001;293(5539):2470-3.

37. Suchan $B$, et al. Reduction of gray matter density in the extrastriate body area in women with anorexia nervosa. Behav Brain Res. 2010;206(1):63-7.

38. Uher $\mathrm{R}$, et al. Functional neuroanatomy of body shape perception in healthy and eating-disordered women. Biol Psychiatry. 2005;58(12):990-7.

39. Suchan B, et al. Reduced connectivity between the left fusiform body area and the extrastriate body area in anorexia nervosa is associated with body image distortion. Behav Brain Res. 2013;241:80-5.

40. Downing PE, et al. The role of the extrastriate body area in action perception. Soc Neurosci. 2006; 1 (1):52-62.

41. Urgesi C, Berlucchi G, Aglioti SM. Magnetic stimulation of extrastriate body area impairs visual processing of nonfacial body parts. Curr Biol. 2004;14(23):2130-4.

42. Carter JC, et al. Relapse in anorexia nervosa: a survival analysis. Psychol Med. 2004;34(4):671-9.

43. Keel PK, et al. Postremission predictors of relapse in women with eating disorders. Am J Psychiatry. 2005;162(12):2263-8.

44. Etkin A, et al. Toward a neurobiology of psychotherapy: basic science and clinical applications. J Neuropsychiatry Clin Neurosci. 2005;17(2):145-58.

45. Nitschke JB, Mackiewicz KL. Prefrontal and anterior cingulate contributions to volition in depression. Int Rev Neurobiol. 2005;67:73-94.

46. Brambilla $F$, et al. Persistent amenorrhoea in weight-recovered anorexics: psychological and biological aspects. Psychiatry Res. 2003;118(3):249-57.

47. Schneider $\mathrm{N}$, et al. Psychopathology in underweight and weight-recovered females with anorexia nervosa. Eat Weight Disord. 2009;14(4):e205-11.

48. Brockhoff $\mathrm{M}$, et al. Cultural differences in body dissatisfaction: Japanese adolescents compared with adolescents from China, Malaysia, Australia, Tonga, and Fiji. Asian J Soc Psychol. 2016;19(4):385-94.

49. Bastiani AM, et al. Perfectionism in anorexia nervosa. Int J Eat Disord. 1995; 17(2):147-52.

50. Sullivan PF, et al. Outcome of anorexia nervosa: a case-control study. Am J Psychiatry. 1998;155(7):939-46.

51. Karlsson GP, Clinton D, Nevonen L. Prediction of weight increase in anorexia nervosa. Nord J Psychiatry. 2013;67(6):424-32.

52. Anderluh MB, et al. Childhood obsessive-compulsive personality traits in adult women with eating disorders: defining a broader eating disorder phenotype. Am J Psychiatry. 2003;160(2):242-7.

53. Fairburn CG, et al. Risk factors for anorexia nervosa: three integrated casecontrol comparisons. Arch Gen Psychiatry. 1999;56(5):468-76.

54. Silver MA, Ress D, Heeger DJ. Neural correlates of sustained spatial attention in human early visual cortex. J Neurophysiol. 2007:97(1):229-37.

55. Ress D, Backus BT, Heeger DJ. Activity in primary visual cortex predicts performance in a visual detection task. Nat Neurosci. 2000;3(9):940-5.

56. Gilon Mann T, et al. Different attention bias patterns in anorexia nervosa restricting and binge/purge types. Eur Eat Disord Rev. 2018;26(4):293-301.

57. Wright Cl, et al. Differential prefrontal cortex and amygdala habituation to repeatedly presented emotional stimuli. Neuroreport. 2001;12(2):379-83.

58. Plichta MM, et al. Amygdala habituation: a reliable fMRI phenotype. Neuroimage. 2014;103:383-90.

59. Wagner $\mathrm{A}$, et al. Normal brain tissue volumes after long-term recovery in anorexia and bulimia nervosa. Biol Psychiatry. 2006;59(3):291-3.

Ready to submit your research? Choose BMC and benefit from:

- fast, convenient online submission

- thorough peer review by experienced researchers in your field

- rapid publication on acceptance

- support for research data, including large and complex data types

- gold Open Access which fosters wider collaboration and increased citations

- maximum visibility for your research: over $100 \mathrm{M}$ website views per year

At BMC, research is always in progress.

Learn more biomedcentral.com/submissions 